\title{
Analysis of the accuracy of actuation electronics in the laser interferometer space antenna pathfinder
}

Cite as: Rev. Sci. Instrum. 91, 045003 (2020); https://doi.org/10.1063/1.5140406

Submitted: 02 December 2019. Accepted: 13 March 2020 . Published Online: 09 April 2020

M. Armano, H. Audley, J. Baird, M. Born, D. Bortoluzzi, (D) N. Cardines, (D) E. Castelli, A. Cavalleri, A. Cesarini, A. M. Cruise, K. Danzmann, M. de Deus Silva, G. Dixon, R. Dolesi, L. Ferraioli, (D) V. Ferroni, E. D. Fitzsimons, M. Freschi, L. Gesa, (D) D. Giardini, F. Gibert, R. Giusteri, C. Grimani, J. Grzymisch, I. Harrison, M.-S. Hartig, G. Heinzel, M. Hewitson, (D) D. Hollington, D. Hoyland, M. Hueller, H. Inchauspé, (D) O. Jennrich, P. Jetzer, (D) N. Karnesis, B. Kaune, C. J. Killow, N. Korsakova, J. P. López-Zaragoza, R. Maarschalkerweerd, D. Mance, V. Martín, L. MartinPolo, J. Martino, F. Martin-Porqueras, I. Mateos, P. W. McNamara, J. Mendes, L. Mendes, N. Meshksar, (D) M. Nofrarias, S. Paczkowski, M. Perreur-Lloyd, A. Petiteau, P. Pivato, (D) E. Plagnol, J. Ramos-Castro, J. Reiche, F. Rivas, (D) D. I. Robertson, G. Russano, J. Slutsky, (D) C. F. Sopuerta, (D) T. Sumner, D. Texier, J. ten Pierick, (D) J. I. Thorpe, (D) D. Vetrugno, S. Vitale, G. Wanner, H. Ward, (D) P. J. Wass, W. J. Weber, L. Wissel, A. Wittchen, and (iD P. Zweifel

\section{ARTICLES YOU MAY BE INTERESTED IN}

Analysis of the accuracy of actuation electronics for the laser interferometer space antenna Review of Scientific Instruments 91, 095003 (2020); https://doi.org/10.1063/5.0018536

Design, fabricate, and experimental verification of an ultrasonic linear motor derived from V-type motors

Review of Scientific Instruments 91, 045002 (2020); https://doi.org/10.1063/1.5129586

Forward modeling of Doppler velocity interferometer system for improved shockwave measurements

Review of Scientific Instruments 91, 043103 (2020); https://doi.org/10.1063/1.5143246

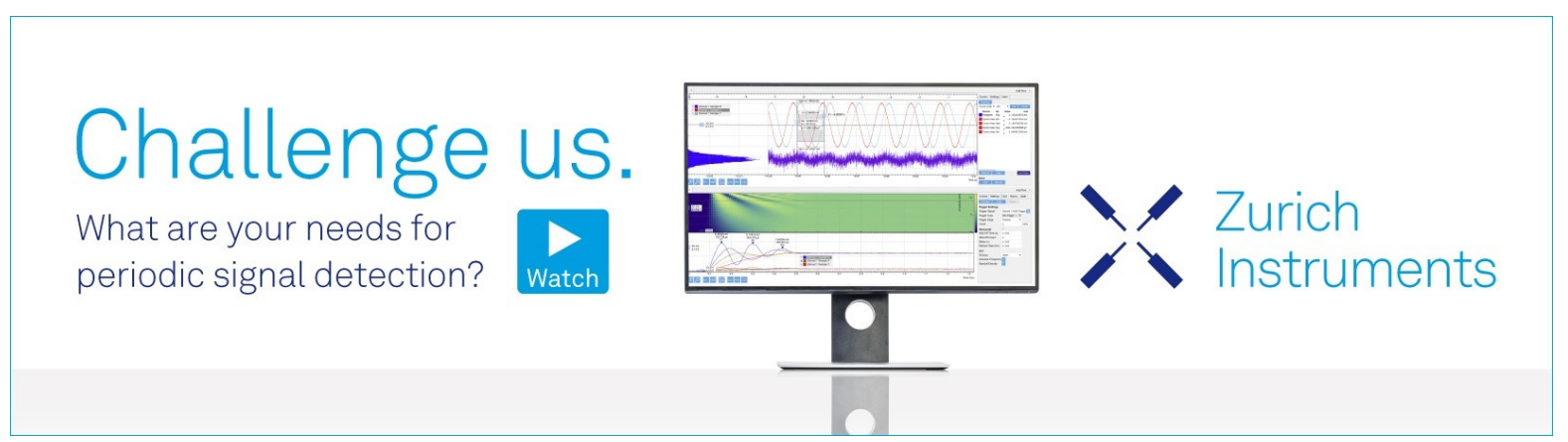




\section{Analysis of the accuracy of actuation electronics in the laser interferometer space antenna pathfinder}

Cite as: Rev. Sci. Instrum. 91, 045003 (2020); doi: 10.1063/1.5140406

Submitted: 2 December 2019 • Accepted: 13 March 2020 •

Published Online: 9 April 2020

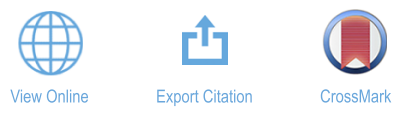

M. Armano, ${ }^{7}$ H. Audley, ${ }^{2}$ J. Baird, ${ }^{3}$ M. Born, ${ }^{2}$ D. Bortoluzzi, ${ }^{4,5}$ N. Cardines, ${ }^{6}$ (D) E. Castelli, ${ }^{5,7}$ (D) A. Cavalleri,
A. Cesarini,
A. M. Cruise 10
K. Danzmann, ${ }^{2}$ M. de Deus Silva,
G. Dixon,
R. Dolesi,
L. Ferraioli,

V. Ferroni, ${ }^{5,7}$

E. D. Fitzsimons, ${ }^{12}$ M. Freschi,

L. Gesa,

D. Giardini,

F. Gibert,

R. Giusteri, ${ }^{2}$

C. Grimani, ${ }^{9}$ J. Grzymisch, ${ }^{1}$ I. Harrison, ${ }^{16}$ M.-S. Hartig, ${ }^{2}$ G. Heinzel, ${ }^{2}$ M. Hewitson, ${ }^{2}$ D. Hollington,

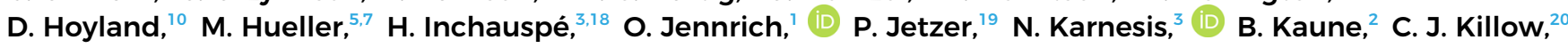

N. Korsakova, ${ }^{21}$ J. P. López-Zaragoza, ${ }^{13}$ R. Maarschalkerweerd, ${ }^{16}$ D. Mance, ${ }^{6}$ V. Martín, ${ }^{13,14}$ L. Martin-Polo, ${ }^{1}$

J. Martino, ${ }^{3}$ F. Martin-Porqueras, ${ }^{11}$ I. Mateos, ${ }^{22}$ P. W. McNamara, ${ }^{1}$ J. Mendes, ${ }^{16}$ L. Mendes, ${ }^{11}$ N. Meshksar, ${ }^{6, b)}$

M. Nofrarias, ${ }^{13,14}$ (D) S. Paczkowski, ${ }^{2}$ M. Perreur-Lloyd, ${ }^{20}$ A. Petiteau, ${ }^{3}$ P. Pivato,,$^{5,7}$ E. Plagnol, ${ }^{3}$

J. Ramos-Castro, ${ }^{14,23}$ J. Reiche, ${ }^{2}$ F. Rivas, ${ }^{13,14}$ D. I. Robertson, ${ }^{20}$ (D) G. Russano, ${ }^{5,7}$ J. Slutsky, ${ }^{24}$ C. F. Sopuerta,

T. Sumner, ${ }^{17}$ (D) D. Texier, ${ }^{11}$ J. ten Pierick, ${ }^{6}$ J. I. Thorpe, ${ }^{24}$ (D) D. Vetrugno, ${ }^{5,7}$ (D) S. Vitale, ${ }^{5,7}$ G. Wanner, ${ }^{2}$ H. Ward, ${ }^{20}$

P. J. Wass, ${ }^{17,18}$ (D) W. J. Weber, ${ }^{5,7}$ L. Wissel, ${ }^{2}$ A. Wittchen, ${ }^{2}$ and P. Zweifel ${ }^{6}$

\footnotetext{
AFFILIATIONS

'European Space Technology Centre, European Space Agency, Keplerlaan 1, 2200 AG Noordwijk, The Netherlands

${ }^{2}$ Albert-Einstein-Institut, Max-Planck-Institut für Gravitationsphysik und Leibniz Universität Hannover, Callinstraße 38, 30167 Hannover, Germany

${ }^{3}$ APC, Univ Paris Diderot, CNRS/IN2P3, CEA/Irfu, Obs de Paris, Sorbonne Paris Cité, 10, rue Alice Domon et Léonie Duquet,

75013 Paris, France

${ }^{4}$ Department of Industrial Engineering, University of Trento, via Sommarive 9, 38123 Trento, Italy

${ }^{5}$ Trento Institute for Fundamental Physics and Application/INFN, 38123 Povo, Trento, Italy

${ }^{6}$ Institut für Geophysik, ETH Zürich, Sonneggstrasse 5, CH-8092 Zürich, Switzerland

${ }^{7}$ Dipartimento di Fisica, Università di Trento, 38123 Povo, Trento, Italy

8Istituto di Fotonica e Nanotecnologie, CNR-Fondazione Bruno Kessler, I-38123 Povo, Trento, Italy

9DISPEA, Università di Urbino "Carlo Bo", Via S. Chiara, 27, 61029 Urbino/INFN, Italy

${ }^{10}$ The School of Physics and Astronomy, University of Birmingham, B15 2TT Birmingham, United Kingdom

"European Space Astronomy Centre, European Space Agency, Villanueva de la Cañada, 28692 Madrid, Spain

${ }^{12}$ The UK Astronomy Technology Centre, Royal Observatory, Edinburgh, Blackford Hill, Edinburgh EH9 3HJ, United Kingdom

${ }^{13}$ Institut de Ciències de l'Espai (ICE, CSIC), Campus UAB, Carrer de Can Magrans s/n, 08193 Cerdanyola del Vallès, Spain

${ }^{14}$ Institut d'Estudis Espacials de Catalunya (IEEC), C/Gran Capità 2-4, 08034 Barcelona, Spain

${ }^{15}$ isardSAT SL, Marie Curie 8-14, 08042 Barcelona, Catalonia, Spain

${ }^{16}$ European Space Operations Centre, European Space Agency, 64293 Darmstadt, Germany

${ }^{17}$ High Energy Physics Group, Physics Department, Imperial College London, Blackett Laboratory, Prince Consort Road, London SW7 2BW, United Kingdom

${ }^{18}$ Department of Mechanical and Aerospace Engineering, MAE-A, University of Florida, P.O. Box 116250, Gainesville,

Florida 32611, USA

${ }^{19}$ Physik Institut, Universität Zürich, Winterthurerstrasse 190, CH-8057 Zürich, Switzerland

${ }^{20}$ SUPA, Institute for Gravitational Research, School of Physics and Astronomy, University of Glasgow,

Glasgow G12 8QQ, United Kingdom
} 


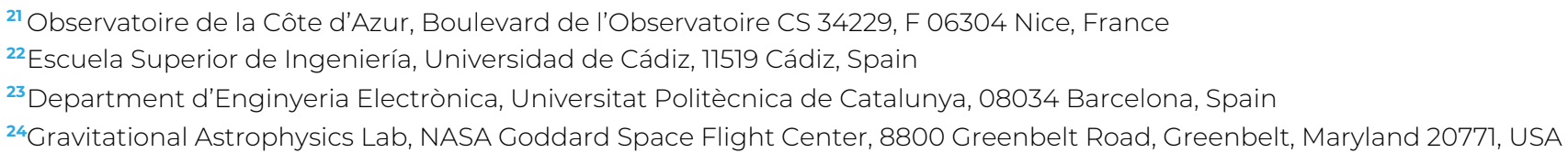

b) Author to whom correspondence should be addressed: neda.meshksar@erdw.ethz.ch

\section{ABSTRACT}

The Laser Interferometer Space Antenna Pathfinder (LPF) main observable, labeled $\Delta g$, is the differential force per unit mass acting on the two test masses under free fall conditions after the contribution of all non-gravitational forces has been compensated. At low frequencies, the differential force is compensated by an applied electrostatic actuation force, which then must be subtracted from the measured acceleration to obtain $\Delta g$. Any inaccuracy in the actuation force contaminates the residual acceleration. This study investigates the accuracy of the electrostatic actuation system and its impact on the LPF main observable. It is shown that the inaccuracy is mainly caused by the rounding errors in the waveform processing and also by the random error caused by the analog to digital converter random noise in the control loop. Both errors are one order of magnitude smaller than the resolution of the commanded voltages. We developed a simulator based on the LPF design to compute the close-to-reality actuation voltages and, consequently, the resulting actuation forces. The simulator is applied during post-processing the LPF data.

Published under license by AIP Publishing. https://doi.org/10.1063/1.5140406

\section{INTRODUCTION}

The Laser Interferometer Space Antenna (LISA) aims to detect gravitational waves in space. The mission was approved by the European Space Agency (ESA) in June 2017, and it is currently in the first planning stages. The technology requirements for the LISA were successfully tested by the LISA Pathfinder ${ }^{2-7}$ (LPF) satellite, which flew from December 2015 to July 2017. In the LPF mission, two test masses (TMs) were put in a nearly perfect free fall condition, and their residual acceleration was measured precisely. Identical TMs will be used in the LISA satellites. Each TM is a gold-platinum cube with $46 \mathrm{~mm}$ edge length surrounded by a set of electrodes in an electrode housing (EH), as shown in Fig. 1. Each electrode creates a capacitor with the TM surface. The electrodes are used to simultaneously sense (electrostatic sensing) and, for all degrees of freedom (DoFs) except the sensitive $x$ interferometer axis, control (electrostatic actuation) the TM position with respect to the center of their EH. Applying a voltage to each electrode induces an electric field and, consequently, an electrostatic control force/torque on the TM. The desired magnitude of the force/torque that should be applied on the TM is calculated by the drag-free attitude control system (DFACS). The DFACS also calculates the analytical conversion of force/torque to voltage. The set of $\mathrm{TM}, \mathrm{EH}$, charge management system, and electronics is called the gravitational reference sensor (GRS). The analog circuits of the GRS front-end electronics (FEE) and the digital code of the field programmable gate array (FPGA) chip inside FEE are responsible for applying the commanded voltages to the corresponding electrodes. The applied voltages are not exactly equal to the commanded voltages because of hardware limitations. The difference between commanded and applied voltages induces a consequent difference between commanded and applied forces/torques, resulting in a loss of actuation accuracy. Quantifying and characterizing this inaccuracy (actuation error) is crucial to understand how this affects the main mission observable of both the LPF and LISA.
In the LPF mission, two GRSs are located in one spacecraft. In science mode, the spacecraft uses micro-Newton thrusters to follow one of the TMs (labeled TM1) along its natural geodesic trajectory. The other TM (labeled TM2) is actuated such that it follows the spacecraft and TM1. The LPF main observable, $\Delta g$, is the total residual acceleration of the two TMs under free fall conditions, along the main measurement axis $x$, and it is calculated by ${ }^{6,}$

$$
\Delta g(t)=\ddot{x}_{12}(t)+\omega_{2}^{2} x_{12}(t)+\left(\omega_{2}^{2}-\omega_{1}^{2}\right) x_{1}(t)-g_{c}(t)-g_{\Omega}(t) .
$$

$\ddot{x}_{12}$ is the second derivative of the relative displacement (relative acceleration) of the TMs, which is measured by using a laser interferometer. $x_{1}$ is the absolute displacement of TM1 with respect to the spacecraft, and it is measured by using a dedicated interferometer. $\omega_{1}^{2}$ and $\omega_{2}^{2}$ are parasitic stiffnesses, and they can be considered as spring constants per unit mass for oscillatory like force couplings between the TMs and the electrode housing. $g_{\Omega}(t)$ is the centrifugal force per unit mass and $g_{c}(t)$ is the actuation force per unit mass applied to TM2 to follow TM1. According to Eq. (1), error in the actuation force along the $x$-axis contaminates the total residual acceleration, $\Delta g$. Thus, it is important to quantify the actuation force error and consider its effect on the calculation of $\Delta g$ for the LPF.

The residual acceleration in the LPF is measured with a precision of $1.74 \pm 0.01 \mathrm{fm} \mathrm{s}^{-2} / \sqrt{\mathrm{Hz}}$ above $2 \mathrm{mHz}$ and $60 \pm 10 \mathrm{fm} \mathrm{s}^{-2} / \sqrt{\mathrm{Hz}}$ at $20 \mu \mathrm{Hz}$. This was achieved, among others, by a more accurate calculation of the electrostatic actuation force based on the method presented in this paper. This paper addresses systematic inaccuracies and an effective correction for these effects in the actuation waveforms. A brief description of the FEE that is important for this study is provided in Sec. II. More details on FEE and how it simultaneously serves for actuation and sensing is given in Refs. 8-11. In order to study the actuation accuracy, we have implemented a simulator that reproduces the "close-to-reality" behavior of the FPGA code and the actuation electronics. The critical elements that cause actuation errors are elaborated in Sec. III, and the simulator 


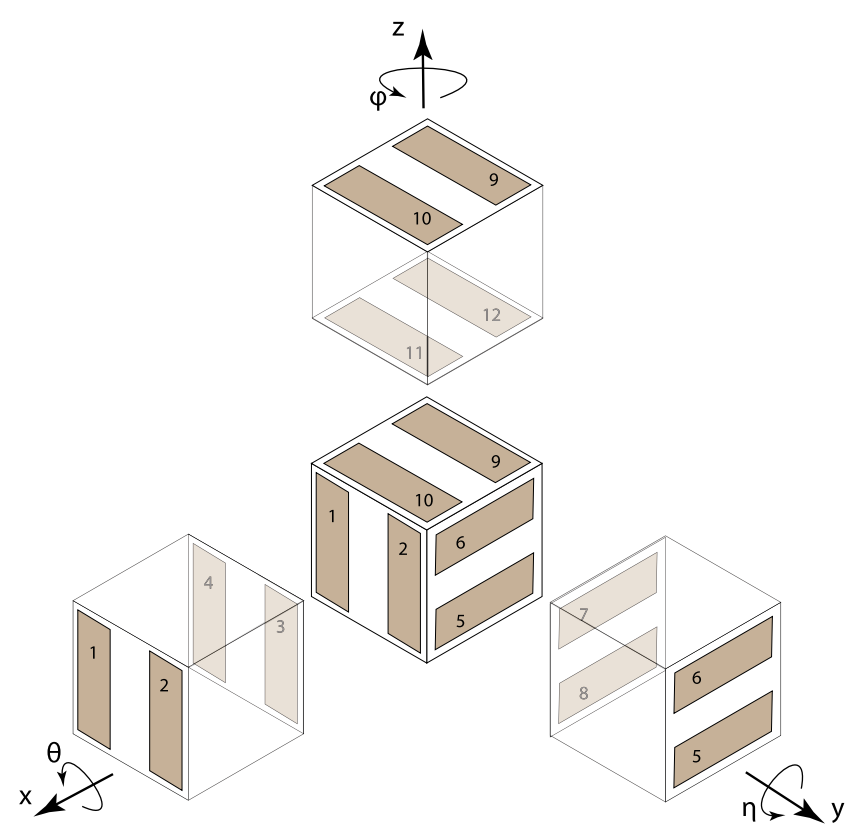

FIG. 1. Electrode arrangement around the TM. The illustrated electrodes are used for TM actuation and position sensing.

is described in Sec. IV. As shown later in the paper, the impact of actuation accuracy on the residual acceleration is especially important at frequencies below $1 \mathrm{mHz}$. Analysis of the actuation error and the impact on LPF data are discussed in Sec. V. A separate article will address the low frequency force noise from slow random fluctuations in the actuators.

\section{ACTUATION ELECTRONICS}

TM actuation is possible in all degrees of freedom (DoFs) by applying commanded force and/or torque to the corresponding electrode. Each electrode is connected to the actuation electronics, which is responsible for exactly two DoFs: one translation and one rotation. Considering Fig. 1, electrodes 1 to 4 are applied for actuation along $x$ and $\varphi$, electrodes 5 to 8 for $y$ and $\theta$, and electrodes 9 to 12 for $z$ and $\eta$.

Figure 2 illustrates the block diagram for actuation along $\mathrm{x}$ and $\varphi$. Actuation along other DoFs is similar. Given the commanded voltage amplitudes for translation and rotation by the DFACS, two orthogonal sinusoidal waveforms (AC signals) are generated, using a common lookup table (LuT). The LuT contains a quarter sinusoid composed of 100 samples, and it is used to make samples at a rate of $12 \mathrm{kHz}$ for the waveforms. The FPGA logic uses the LuT to generate waveforms at a frequency of $n \times 30 \mathrm{~Hz}$. For the waveforms at those frequencies, each sample can be calculated by using an entry of the same LuT, where the frequency depends on how the entries are picked. The integer $n$ is set differently for each DoF such that the generated waveforms are all orthogonal to each other and no cross talk occurs. This yields $60 \mathrm{~Hz}$ and $270 \mathrm{~Hz}$ for actuation along $x$ and $\varphi, 90 \mathrm{~Hz}$ and $240 \mathrm{~Hz}$ for $\mathrm{y}$ and $\theta$, and $120 \mathrm{~Hz}$ and $180 \mathrm{~Hz}$ for $\mathrm{z}$ and $\eta$. The amplitudes of the generated sinusoidal waveforms, labeled $V_{\text {cmd }}$, are commanded by the DFACS at $10 \mathrm{~Hz}$ frequency. Thus, the waveforms are applied for $100 \mathrm{~ms}$ that form integer multiplications of the waveform periods. The commanded voltage amplitudes are discretized with a resolution of $153 \mu \mathrm{V}$, described in this paper as the least significant bit (LSB) voltage. In addition to these AC signals, DC voltages, labeled $V_{\mathrm{cmd}, \mathrm{DC}}$, can also be commanded by the DFACS to compensate for parasitic electrode voltages and to aid TM charge measurement and discharge. The AC and DC signals are combined as given in Eq. (2).

After combining the waveforms, the combined signal is mapped from the LSB value used for the commanded voltage $\left( \pm 10 \mathrm{~V} / 2^{17} \simeq 15, \mu \mathrm{V}\right)$ to the one used for the proportional, integral, and derivative (PID) controller $\left( \pm 14.5 \mathrm{~V} / 2^{18} \simeq 111 \mu \mathrm{V}\right)$. Thus, the LSB value of the control loop is a factor of 1.379 smaller. The PID controller was designed for a different LSB value in order to make use of the full conversion range of the analog to digital converter (ADC) used for its feedback, i.e., the signal is scaled to use the full range of the ADC output codes. This was done to minimize the noise contribution of the ADC, which has an input inferred noise of $0.72 \mathrm{LSB}_{\mathrm{RMS}}$, according to the data sheet for the ADC component LTC1604. ${ }^{13}$ To maintain the same amplitude, the digital values are multiplied by the reciprocal factor before entering the loop. For that, they are multiplied by the factor 1.375 , which is the closest value to 1.379 that can be represented with three fractional bits, and it is well within the tolerance of the analog gain, for which a calibration is performed. This is shown in Figs. 2 and 3 by the "Scale 1.375" subblocks. As discussed later in this paper, scaling the waveform, as it is currently designed in the LPF, causes an actuation error, which cannot be neglected. The current electronics design requires several optimizations, before it can be integrated in the LISA. These are proposed in a separate study. ${ }^{1 .}$

In order to increase the signal resolution and reduce the actuation error, the $12 \mathrm{kHz}$ sampled signal is up-sampled to $96 \mathrm{kHz}$ by linear interpolation and then fed to a sigma-delta control loop. The control loop consists of a PID controller with the derivative block configured feed-forward and the proportional and integral blocks acting on the PID error signal. To generate an analog signal at the electrode, a digital-to-analog converter (DAC) is used, followed by an analog integrator, which has an integration gain that fits with the feed-forward gain of the digital part. Finally, to provide the feedback for the PID controller, the signal is digitized by an ADC.

The signals injected to electrodes 1 to 4 are then given by

$V_{1}(t)=+V_{\mathrm{cmd}, 1 x} \sin (2 \pi 60 t)+V_{\mathrm{cmd}, 1 \varphi} \sin (2 \pi 270 t)+V_{\mathrm{cmd}, \mathrm{DC} 1}$,

$V_{2}(t)=-V_{\mathrm{cmd}, 1 x} \sin (2 \pi 60 t)+V_{\mathrm{cmd}, 2 \varphi} \cos (2 \pi 270 t)+V_{\mathrm{cmd}, \mathrm{DC} 2}$,

$V_{3}(t)=+V_{\mathrm{cmd}, 2 x} \cos (2 \pi 60 t)-V_{\mathrm{cmd}, 1 \varphi} \sin (2 \pi 270 t)+V_{\mathrm{cmd}, \mathrm{DC} 3}$,

$V_{4}(t)=-V_{\mathrm{cmd}, 2 x} \cos (2 \pi 60 t)-V_{\mathrm{cmd}, 2 \varphi} \cos (2 \pi 270 t)+V_{\mathrm{cmd}, \mathrm{DC} 4}$.

The time-averaged $x$-force and $\varphi$-torque applied to the TM are calculated by

$$
\begin{aligned}
& F_{x}=\frac{1}{2}\left(\left|\frac{\partial C_{E L, T M}}{\partial x}\right|-\left|\frac{\partial C_{E L, H}}{\partial x}\right|\right)\left(\left\langle V_{1}^{2}\right\rangle+\left\langle V_{2}^{2}\right\rangle-\left\langle V_{3}^{2}\right\rangle-\left\langle V_{4}^{2}\right\rangle\right), \\
& T_{\varphi}=\frac{1}{2}\left(\left|\frac{\partial C_{E L, T M}}{\partial \varphi}\right|-\left|\frac{\partial C_{E L, H}}{\partial \varphi}\right|\right)\left(\left\langle V_{1}^{2}\right\rangle-\left\langle V_{2}^{2}\right\rangle+\left\langle V_{3}^{2}\right\rangle-\left\langle V_{4}^{2}\right\rangle\right),
\end{aligned}
$$

with $C_{E L, T M}$ being the capacitance between each electrode and the TM and $C_{E L, H}$ being the one between the electrode and the housing, respectively. 


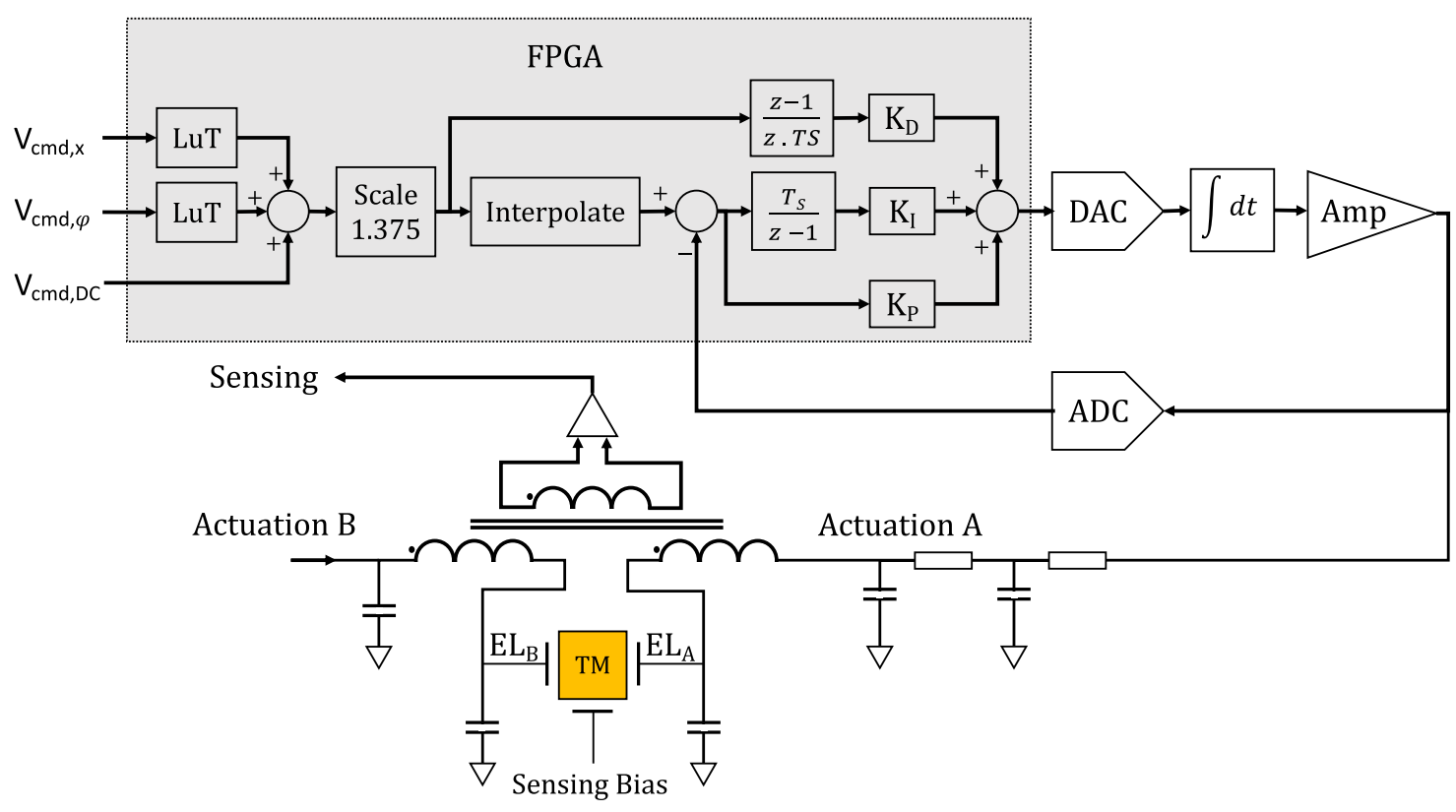

FIG. 2. Circuit block diagram featuring actuation elements for two opposing electrodes. Here, the commanded voltages correspond to $x / \varphi$ actuation. Actuation along other DoFs is similar.

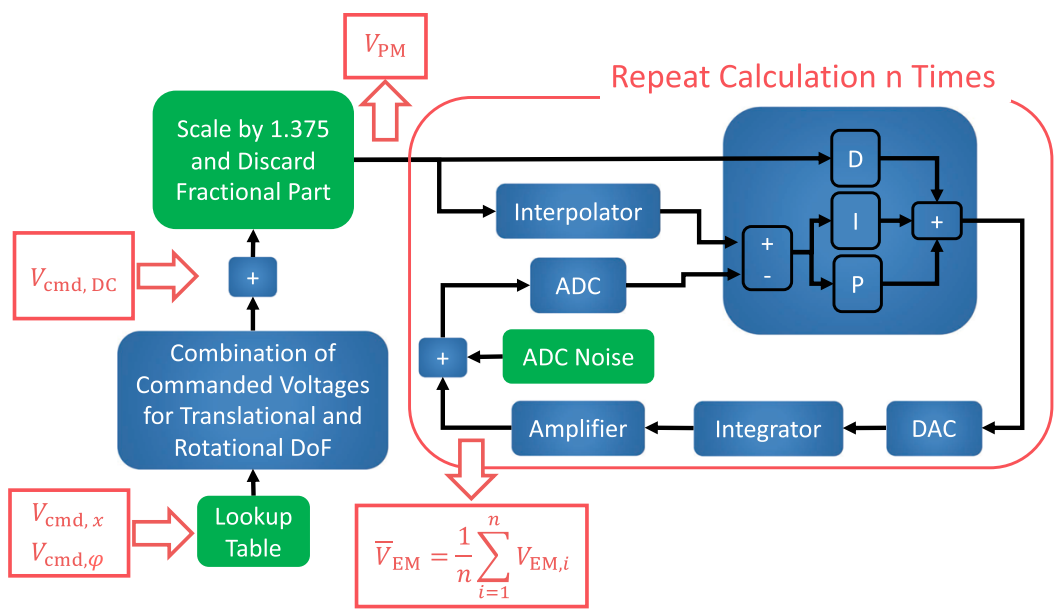

FIG. 3. Simulation scheme. $V_{c m d}$ and $V_{c m d, D C}$ are the inputs. $V_{\mathrm{PM}}$ and $\bar{V}_{\mathrm{EM}}$ are the outputs of the PM and EM, respectively.

\section{ACTUATION ACCURACY}

The accuracy of the actuation system is mainly affected by rounding errors and also by the random noise caused by the ADC in the control loop. These are elaborated below.

\section{- Number of available bits in waveform generation}

The AC commanded voltages from the DFACS are 16 bit unsigned integers with a $153 \mu \mathrm{V}$ resolution. These are multiplied by LuT words, which are 16 bit integers. The multiplication would result in 32 bit integers, whereas only 16 bits are used in the FPGA code. Therefore, the lower significant 16 bits are discarded and the generated waveform is rounded to 16 bits, and this introduces a rounding error.
While a change in 1 LSB in the commanded AC voltage will, indeed, change the amplitude of the sinusoidal waveform (peak voltage) by $1 \mathrm{LSB}$, the rest of the points in the waveform will change by 0 or $1 \mathrm{LSB}$, depending on the rounding. This results in changing the effective voltage on the electrode [see Eq. (4)] by an amount around, but not exactly, $1 \mathrm{LSB}$, and it directly influences the applied force/torque, according to Eq. (3).

\section{- Scaling after waveform generation}

After scaling by the non-integer factor of 1.375 , the fractional part is removed. Similar to the last step, this also results in a rounding error. Data resolution at this stage is $153 \mu \mathrm{V} / 1.375=111 \mu \mathrm{V}$. 


\section{- Error caused by ADC random noise}

Among the electronic elements used in the actuation system, DAC and ADC produce the major noise. Although the noise entering the feedback loop is compensated, the noise caused by the ADC is critical, because it directly affects the behavior of the loop. Simulation of the ADC noise is elaborated in Appendix A.

The first two points describe the rounding error that happens on single samples of a waveform. This error is one order of magnitude smaller than the resolution of the commanded voltages, as elaborated in Sec. V A and illustrated in Fig. 5. The random error caused by ADC random noise is also one order of magnitude smaller than the resolution of the commanded voltages. This is discussed in Sec V B and illustrated in Fig. 6.

\section{SIMULATION}

We implemented a simulation of the actuation system based on the LPF design, in order to reproduce the close-to-reality actuation voltages and, consequently, actuation forces/torques. The critical elements affecting the actuation accuracy were mentioned in Sec. III. They are implemented in the simulation in order to quantify their impact. The simulation is divided into two main parts: The first simulates only the waveform generation and scaling and essentially considers the systematic error between the perfect analog sinusoidal wave and the digitized generated waveform. We refer to this part as a partial model (PM). The second part simulates the entire circuitry, which includes the waveform generation, scaling, digital controller, and feedback loop. We call this part entire model (EM). The EM allows us to investigate the effect of ADC random noise on actuation accuracy and also the systematic error of the control loop.

The simulation inputs are the commanded (AC) voltages $V_{\mathrm{cmd}}$, which are the peak amplitudes of the generated waveforms, and also the commanded DC voltage $V_{\text {cmd,DC. The sinusoidal wave- }}$ forms $V_{i}(t)$ applied to each electrode $i$ are implemented according to Eq. (2), with $i$ denoting electrodes 1 to 4 , which are responsible for $x / \varphi$ actuation. The effective voltage on each electrode is the root mean square (rms) of $V_{i}(t)$. The rate of DFACS commanded voltages is $10 \mathrm{~Hz}$ and the waveforms are $100 \mathrm{~ms}$ long. The simulation outputs $V_{\text {out }, x, i}$ and $V_{\text {out }, \varphi, i}$ are the amplitudes of the AC signal (peak voltages) at $60 \mathrm{~Hz}$ and $270 \mathrm{~Hz}$ frequencies, which are numerically evaluated in the simulation by the fast Fourier transform of the waveform $V_{i}(t)$ applied to electrode $i$. The effective voltage on each electrode is related to the peak voltages as follows:

$$
\operatorname{RMS}\left(V_{i}(t)\right)=\sqrt{\frac{1}{2}\left(V_{\text {out } x, i}^{2}+V_{\text {out }, \varphi, i}^{2}\right)}, i=1, \ldots, 4 .
$$

For each input command voltage, $V_{\text {out }, x, i}$ and $V_{\text {out }, \varphi, i}$ are calculated by both the PM and EM. For the sake of simplicity, only the actuation of TM2 along $x$ is discussed further in this paper, and the applied voltages to electrode 1 are illustrated. Therefore, the indices $x$ and $i=1$ are removed, and the simulation output is labeled $V_{\mathrm{PM}}$ or $V_{\mathrm{EM}}$, with respect to $\mathrm{PM}$ and EM. The analysis for other electrodes and also other DoFs provide similar results. Figure 3 illustrates the simulation scheme.

\section{ANALYSIS AND RESULTS}

In this section, we elaborate on the analyses related to quantifying the systematic error of the waveform generation, the systematic error of the control loop, and also the random error of the control loop. The systematic error of the waveform generation is studied by comparing $V_{\mathrm{PM}}$ to the commanded voltages, and the systematic and also the random errors of the control loop are investigated by comparing $V_{\mathrm{EM}}$ to $V_{\mathrm{PM}}$.

For these analyses, the simulation input is an integer multiplication of the LSB value, given by $V_{\text {cmd }}=153 \mu \mathrm{V} \cdot \mathfrak{D}_{c m d}$, whereby $\mathfrak{D}_{c m d}$ denotes the integer value of the digital code resulting from a commanded voltage. $V_{\mathrm{cmd}, \mathrm{DC}}$ is zero. We consider $V_{\mathrm{cmd}}$ from $153 \mathrm{mV}$ to $535 \mathrm{mV}$, which corresponds to the digital values of $\mathfrak{D}_{c m d}$ from 1000 to 3500 . Voltages around $500 \mathrm{mV}$ are especially interesting, because they correspond to the LPF commands during the acceleration measurements.

At the end of this section, we analyze the effect of the actuation error on the LPF data. For this analysis, the simulation
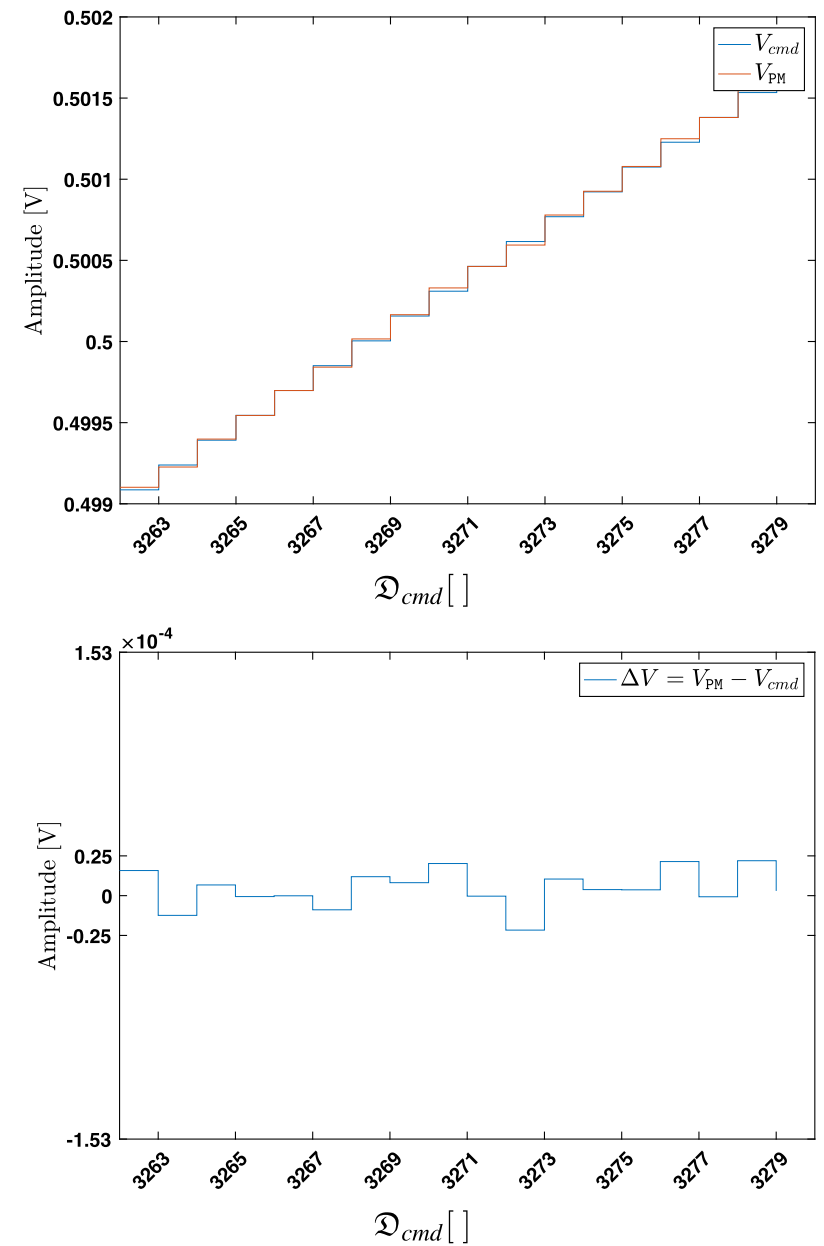

FIG. 4. Comparing $V_{\text {cmd }}$ to $V_{P M}$ shows that the resolution of $V_{P M}$ varies for differen input commands. The variation is one order of magnitude smaller than that of the $V_{\text {cmd }}$ resolution, $153 \mu \mathrm{V}$. 
inputs are the commanded voltages for several LPF acceleration measurements.

\section{A. Systematic error of the waveform generation}

In Fig. $4, V_{\mathrm{cmd}}$ is compared to $V_{\mathrm{PM}}$. The resolution of $V_{\mathrm{cmd}}$ (vertical difference between adjacent steps) is equal to $153 \mu \mathrm{V}$. For $V_{\mathrm{PM}}$, however, the resolution varies for different digital commands. This variation is due to the rounding error after the waveform generation and also after the waveform scaling. As shown in Fig. 5, $\Delta V$ $=V_{\mathrm{PM}}-V_{\mathrm{cmd}}$ is typically of the order of $10 \mu \mathrm{V}$, which is one order of magnitude smaller than that of the input resolution. Considering Eq. (3), $\Delta V$ in average introduces an erroneous induced field and results in a force noise.

\section{B. Error of the control loop}

The ADC random noise is a white noise, and it dominates the systematic error of the control loop. Therefore, the simulation is implemented such that it allows suppressing the random noise, in order to quantify the systematic error of the control loop. To this purpose, the simulator repeats the calculation of $V_{\mathrm{EM}}$ (usually $n=10^{4}, 10^{5}$, or $10^{6}$ times) and outputs the averaged value $\bar{V}_{\mathrm{EM}}=\frac{1}{n} \sum_{i=1}^{n} V_{\mathrm{EM}, i}$.

A fit of the data from simulation outputs $\bar{V}_{\mathrm{EM}}$ and $V_{\mathrm{PM}}$ demonstrates a relation $\bar{V}_{\mathrm{EM}}=\alpha V_{\mathrm{PM}}+\beta$. The values $\alpha$ and $\beta$ are different for various $n$ values. The error of the control loop is specified by $\Delta V_{n}=\bar{V}_{\mathrm{EM}}-\left(\alpha V_{\mathrm{PM}}+\beta\right)$.

The random error is determined by $\Delta V_{n=1}$, and it is illustrated in Fig. 6. The systematic error is verified by $\Delta V_{n=10^{6}}$, and it is shown in Fig. 7. In Appendix B, we elaborate why $n=10^{6}$ is sufficient for analyzing the systematic error. The systematic error is of the order of $10^{-8} \mathrm{~V}$, and its effect on the accuracy is negligible. It is at worse four orders of magnitude smaller than the resolution of the commanded voltages and, thus, 1000 times smaller than the systematic rounding error (see Figs. 4 and 5).

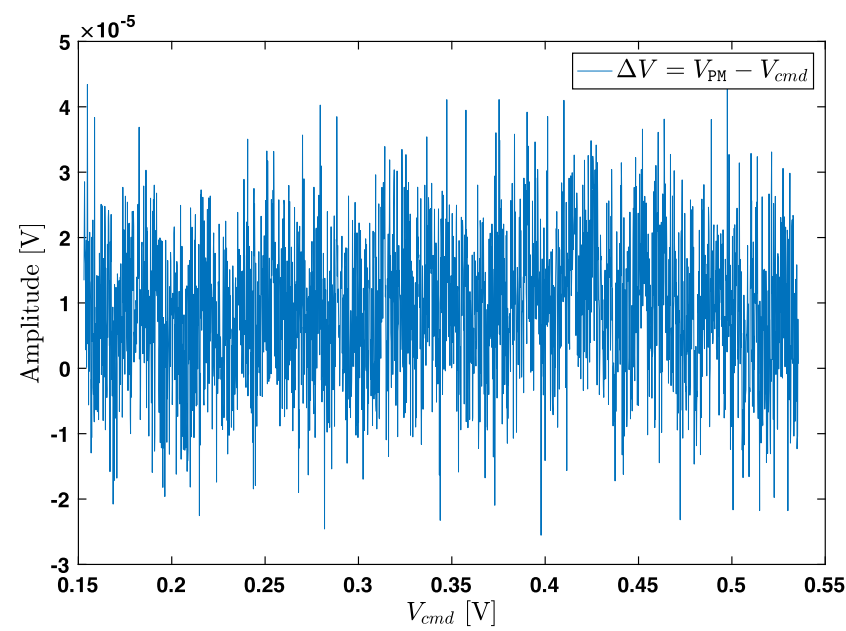

FIG. 5. The variation of the $V_{P M}$ resolution is one order of magnitude smaller than the resolution of commanded voltages, $153 \mu \mathrm{V}$. Thus, typical amplitude errors are of order 0.1 LSB.

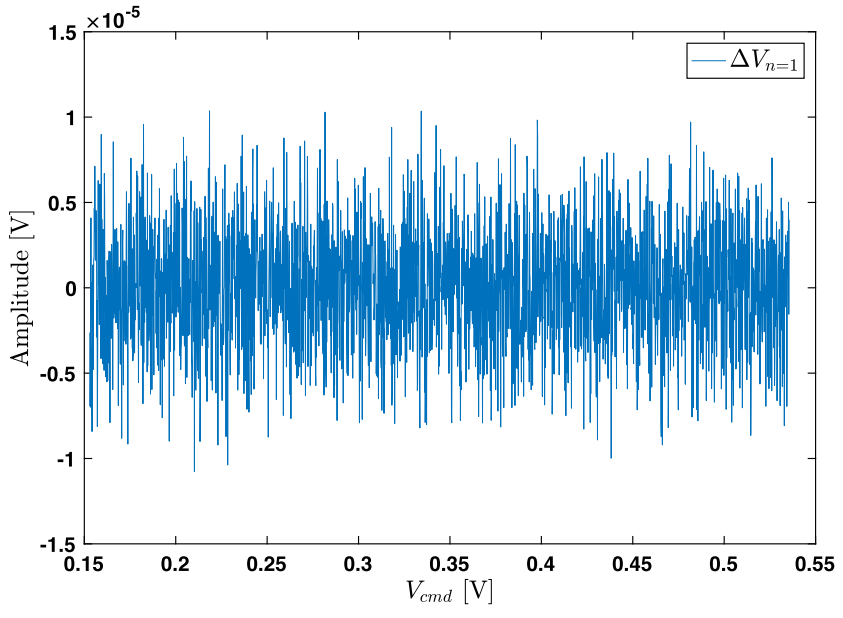

FIG. 6. The figure illustrates $\Delta V_{n=1}=V_{\mathrm{EM}}-\left(0.73 V_{\mathrm{PM}}-9 \mathrm{nV}\right)$. Note that here, $\bar{V}_{\mathrm{EM}}=V_{\mathrm{EM}}$, as $n=1$. The random error caused by the ADC random noise is of the order of $10 \mu \mathrm{V}$.

\section{Effect of the actuation error on the LPF data}

The LPF main observable, $\Delta g$, is the residual acceleration of the two test masses, and it is calculated by Eq. (1). According to this equation, the error of the actuation force per unit mass, $g_{c}(t)$, contaminates $\Delta g$, especially at frequencies below $1 \mathrm{mHz}{ }^{6}$ In order to investigate the effect of actuation inaccuracy on the LPF data, we first calculated the actuation force by $V_{\mathrm{cmd}}, V_{\mathrm{PM}}$, and $\bar{V}_{\mathrm{EM}}$, respectively, and afterward, we considered the amplitude spectral density of the residual acceleration, $S_{\Delta g}^{1 / 2}(f)$, at a low frequency, i.e., $f=0.1$ $\mathrm{mHz}$, for the LPF acceleration measurements. As shown in Fig. 8, calculation of the actuation force by $V_{\mathrm{PM}}$ (red curve) results in a lower amplitude spectral density, $S_{\Delta g}^{1 / 2}(f=0.1 \mathrm{mHz})$, compared to

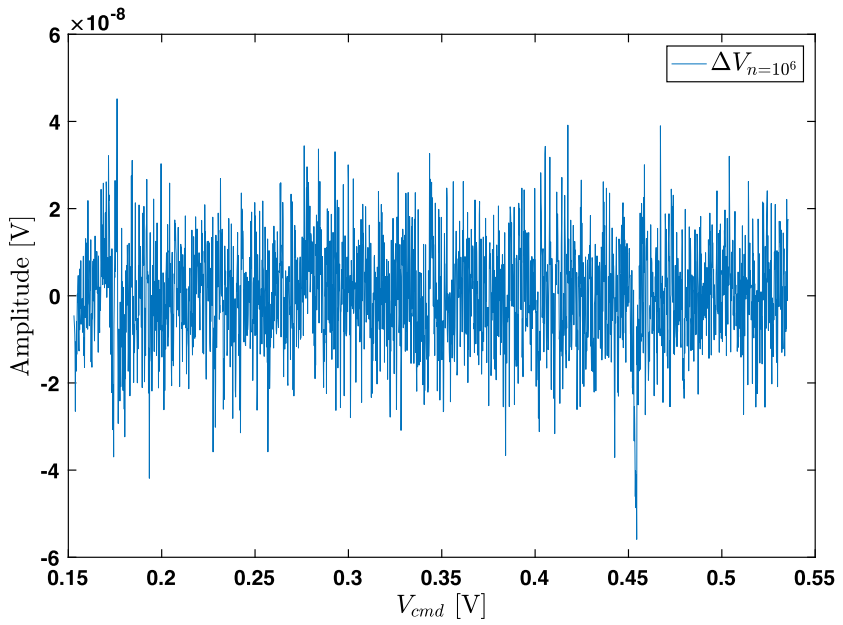

FIG. 7. The error of the control loop $\Delta V_{n=10^{6}}=\bar{V}_{\mathrm{EM}}-\left(1.03 V_{\mathrm{PM}}-2.7 \mathrm{nV}\right)$ is four orders of magnitude smaller than the resolution of commanded voltages, $153 \mu \mathrm{V}$. 


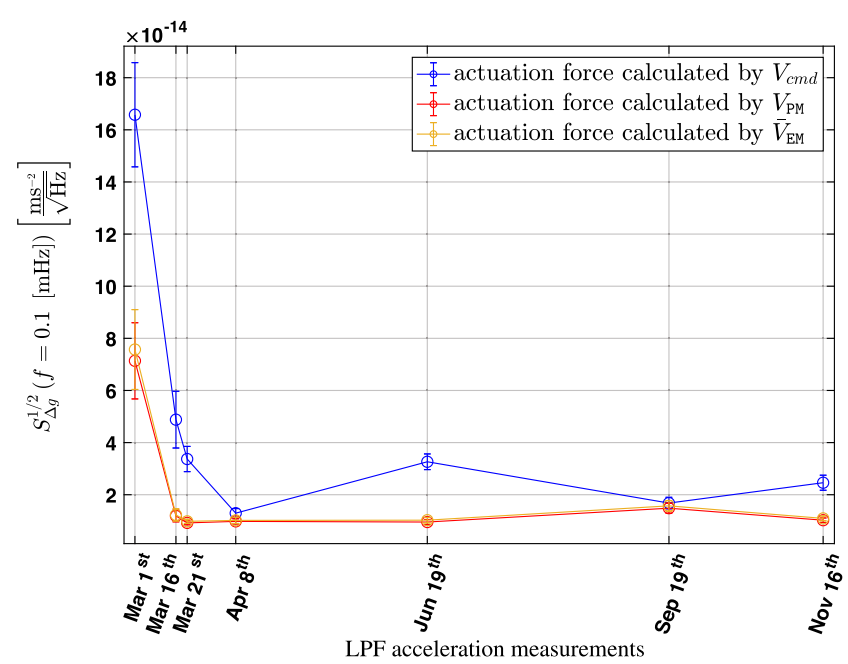

FIG. 8. Amplitude spectral density of the LPF residual test-mass acceleration at $0.1 \mathrm{mHz}$ for different segments of acceleration measurement in 2016. Considering the actuation inaccuracy in the calculation of $\Delta g$ results in a stationary amplitude spectral density $S_{\Delta g}^{1 / 2}$ at $f=0.1 \mathrm{mHz}$ frequency.

the case in which the actuation force is calculated by $V_{\text {cmd }}$ (blue curve).

Comparing the data calculated by $V_{\mathrm{PM}}$ (red curve) to the ones calculated by $\bar{V}_{\mathrm{EM}}$ (yellow curve) indicates that the governing actuation error refers to the error caused by rounding and scaling the waveform and not the error of the control loop. This is also illustrated in Fig. 9, where the amplitude spectral density of $\Delta g$ is calculated for the LPF acceleration measurement in June 19th. As shown in the figure, considering the inaccuracy in the calculation of $\Delta g$ is especially important at low frequencies.

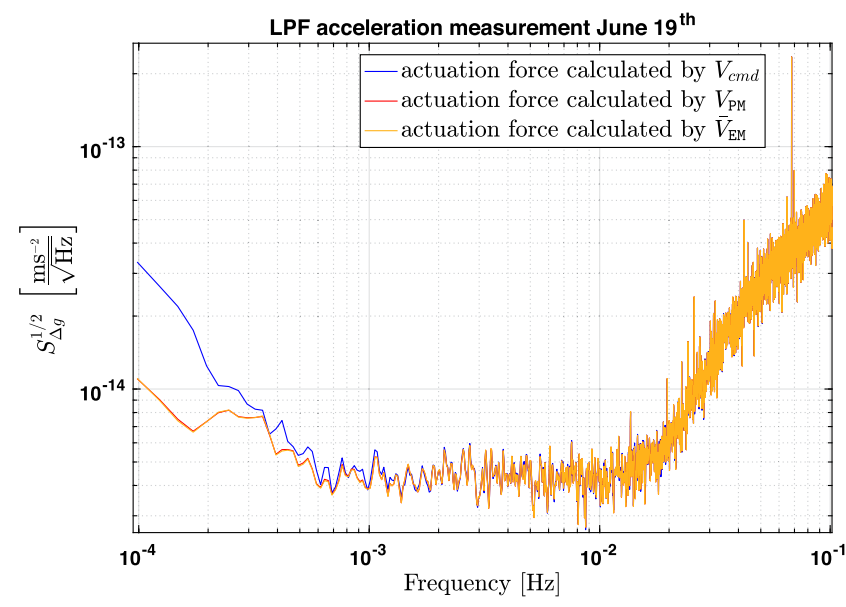

FIG. 9. Amplitude spectral density of the LPF residual test-mass acceleration for the measurement in June 2016.

\section{CONCLUSION}

We have identified how the systematic rounding errors have a relevant and detectable impact on the AC actuation amplitudes and developed a simulator to verify the expected rounding behavior. Using the simulation results, we have also quantified the random error of the control loop, which is caused by the $\mathrm{ADC}$ random noise. Both these errors are of the order of $10 \mu \mathrm{V}$, which is one order of magnitude smaller than the resolution of the input commanded voltages $(153 \mu \mathrm{V})$. We also analyzed the systematic error of the control loop by suppressing the ADC random noise by three orders of magnitude in the simulation. It was shown that this error is four orders of magnitude smaller than the resolution of the commanded voltages and, thus, negligible.

Considering the systematic rounding errors, our simulator enables us to estimate the close-to-reality value of the actuation forces and more accurately calculate the residual acceleration of the test masses, which is the LPF main observable, at low frequencies. It is worth mentioning that actuation forces are calculated by our simulator in post-processing the LPF data.

Contrary to the LPF, there is no actuation along the main measurement axis in the LISA. However, optimizing the electronics to avoid the systematic error, especially in waveform generation, is relevant for accurate actuation of the TMs in other DoFs. A proposal for optimizing the waveform generation for the LISA will be provided in a separate study. ${ }^{14}$

\section{ACKNOWLEDGMENTS}

This work was supported by ETH Research Grant No. ETH-05 16-2, and it has been made possible by the LISA Pathfinder mission, which is part of the space-science program of the European Space Agency.

The French contribution has been supported by the CNES (Accord Specific de Projet Grant No. CNES 1316634/CNRS 103747), the CNRS, the Observatoire de Paris, and University Paris-Diderot. E. Plagnol and H. Inchauspé would also like to acknowledge the financial support of the UnivEarthS Labex program at Sorbonne Paris Cité (Grant Nos. ANR-10-LABX-0023 and ANR-11-IDEX0005-02).

The Albert-Einstein-Institut acknowledges the support of the German Space Agency, DLR. The work is supported by the Federal Ministry for Economic Affairs and Energy based on a resolution of the German Bundestag (Grant Nos. FKZ 50OQ0501 and FKZ 50OQ1601).

The Italian contribution has been supported by the Agenzia Spaziale Italiana and the Istituto Nazionale di Fisica Nucleare.

The Spanish contribution has been supported by Contract Nos. AYA2010-15709 (MICINN), ESP2013-47637-P, ESP2015-67234-P, and ESP2017-90084-P (MINECO). Support from AGAUR (Generalitat de Catalunya) contract 2017-SGR-1469 is also acknowledged. M. Nofrarias acknowledges support from Fundacion General CSIC (Programa ComFuturo). F. Rivas acknowledges an FPI contract from MINECO.

The Swiss contribution acknowledges the support of the Swiss Space Office (SSO) via the PRODEX Programme of the ESA. L. Ferraioli is supported by the Swiss National Science Foundation. 
The UK groups wish to acknowledge support from the United Kingdom Space Agency (UKSA), the University of Glasgow, the University of Birmingham, Imperial College, and the Scottish Universities Physics Alliance (SUPA).

J. I. Thorpe and J. Slutsky acknowledge the support of the U.S. National Aeronautics and Space Administration (NASA).

$\mathrm{N}$. Korsakova would like to thank the support from the CNES Fellowship.

The LISA Pathfinder collaboration would like to acknowledge Professor Pierre Binetruy (deceased 30 March 2017) and Professor José Alberto Lobo (deceased 30 September 2012) for their contribution to the LISA Pathfinder science.

\section{APPENDIX A: ADC NOISE}

The ADC noise density is calculated from the signal-to-noise ratio (SNR), which is $90.1 \mathrm{~dB}$ according to the data sheet for the ADC component LTC1604,

$$
\mathrm{SNR}=90.1 \mathrm{~dB}=20 \log \left(\frac{\mathrm{RMS}_{\text {signal }}}{\mathrm{RMS}_{\text {noise }}}\right) .
$$

The rms value for the signal at the ADC input is calculated from the amplitude of the generated sinusoidal signal $\left(\mathrm{A}_{\text {signal }}\right)$, and it is given by

$$
\mathrm{RMS}_{\text {signal }}=\frac{\mathrm{A}_{\text {signal }}}{\sqrt{2}}=\frac{20 \mathrm{~V} / 1.375}{\sqrt{2}}=10.2852 \mathrm{~V}_{\mathrm{RMS}} .
$$

$20 \mathrm{~V}$ refers to the maximum commanded AC amplitude for each actuation channel, and the scaling factor is elaborated in Sec. II. Therefore, $\mathrm{RMS}_{\text {noise }}=321.52 \mu \mathrm{V}_{\mathrm{RMS}}$ is obtained by substituting Eq. (A2) in (A1) and, it corresponds to $\mathrm{RMS}_{\text {noise }}=0.72 \mathrm{LSB}$ given in the data sheet. The noise density is then given by

$$
S_{\text {ADC }}^{1 / 2}=\frac{\mathrm{RMS}_{\text {noise }}}{\sqrt{f_{s}}}=1.0377 \frac{\mu \mathrm{V}}{\sqrt{\mathrm{Hz}}},
$$

whereby $f_{s}=96 \mathrm{kHz}$ is the sampling frequency of the signal. The implemented noise in the simulation is a pseudorandom white noise drawn from the normal distribution with zero mean and standard deviation $\sigma_{\mathrm{ADC}}=\mathrm{RMS}_{\text {noise }}=321.52 \mu \mathrm{V}$.

For big number of iterations, such as $n=10^{5}$ and $10^{6}$, the output of the EM is parallelly computed. In order to avoid the same random numbers generated by different processor cores, the random number generation is seeded, using the Matlab function $\operatorname{rng}($ seed).

\section{APPENDIX B: CROSS CORRELATION}

The random error of the control loop is labeled $\Delta V_{1}$, and its standard deviation is $\sigma_{\Delta V_{1}}=3.5 \mu \mathrm{V}$ for the commanded voltages 0.153-0.536 V. These data are shown in Fig. 6.

According to the central limit theorem, we expect that the random error is reduced by $\sigma_{\Delta V_{1}} / \sqrt{10^{6}}=3.5 \mathrm{nV}$ for $n=10^{6}$ iterations. However, the standard deviation of $\Delta V_{10^{6}}$ obtained by the simulation is $\sigma_{\Delta V_{10^{6}}}=11.8 \mathrm{nV}\left(\Delta V_{10^{6}}\right.$ data are illustrated in Fig. 7). It means that $\sigma_{\Delta V_{10^{6}}}$ derived from the simulated data is 3.37 times bigger than the value expected from the central limit theorem. Therefore, $\Delta V_{10^{6}}$ is not purely random, and it is dominated by the systematic error.

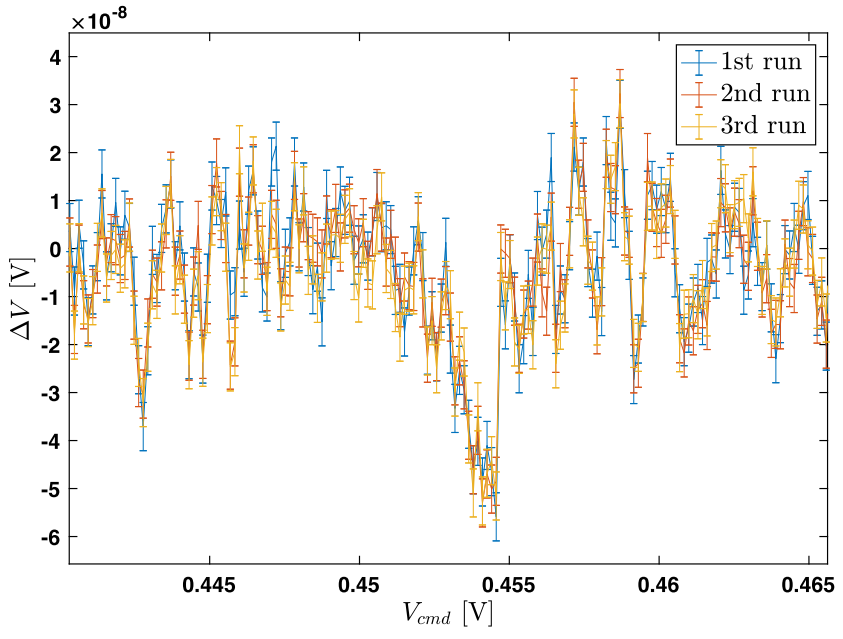

FIG. 10. $\Delta V=\bar{V}_{\mathrm{EM}}-V_{\mathrm{PM}, \mathrm{fitted}}$ is calculated three times. The parallel trajectories indicate a systematic error in the control loop.

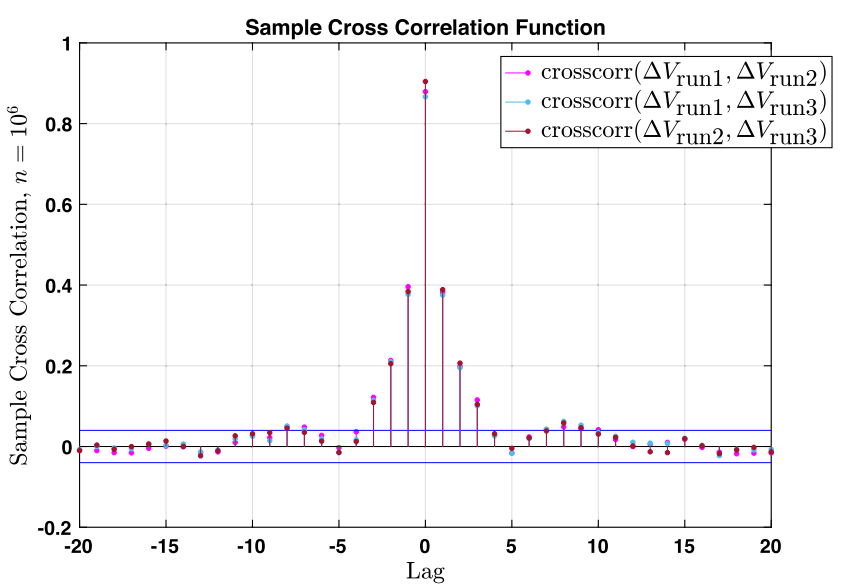

FIG. 11. The sample cross correlation of each two runs at lag zero proves the systematic error in the control loop.

This is also analyzed by the sample cross correlation function, as described below.

For the same input voltages, we calculated $\Delta V_{n=10^{6}}$ three times and labeled each calculation a run. The index $n=10^{6}$ indicates that each $\bar{V}_{\mathrm{EM}}$ is averaged over $10^{6}$ iterations. As shown in Fig. 10, the three runs follow similar trajectories. Furthermore, the sample cross correlation coefficient of the combination of pairs of runs at lag zero is close to one, as illustrated in Fig. 11. This proves the dependency of $\Delta V_{n=10^{6}}$ on the commanded voltages and indicates a systematic error in the control loop.

\section{REFERENCES}

${ }^{1}$ P. Amaro-Seoane, H. Audley, S. Babak, J. Baker, E. Barausse, P. Bender, E. Berti, P. Binetruy, M. Born, D. Bortoluzzi, J. Camp, C. Caprini, V. Cardoso, M. Colpi, J. Conklin, N. Cornish, C. Cutler, K. Danzmann, R. Dolesi, L. Ferraioli, V. Ferroni, E. Fitzsimons, J. Gair, L. G. Bote, D. Giardini, F. Gibert, C. Grimani, 
H. Halloin, G. Heinzel, T. Hertog, M. Hewitson, K. Holley-Bockelmann, D. Hollington, M. Hueller, H. Inchauspe, P. Jetzer, N. Karnesis, C. Killow, A. Klein, B. Klipstein, N. Korsakova, S. L. Larson, J. Livas, I. Lloro, N. Man, D. Mance, J. Martino, I. Mateos, K. McKenzie, S. T. McWilliams, C. Miller, G. Mueller, G. Nardini, G. Nelemans, M. Nofrarias, A. Petiteau, P. Pivato, E. Plagnol, E. Porter, J. Reiche, D. Robertson, N. Robertson, E. Rossi, G. Russano, B. Schutz, A. Sesana, D. Shoemaker, J. Slutsky, C. F. Sopuerta, T. Sumner, N. Tamanini, I. Thorpe, M. Troebs, M. Vallisneri, A. Vecchio, D. Vetrugno, S. Vitale, M. Volonteri, G. Wanner, H. Ward, P. Wass, W. Weber, J. Ziemer, and P. Zweifel, "Laser interferometer space antenna," arXiv:1702.00786 (2017).

${ }^{2}$ P. McNamara, S. Vitale, and K. Danzmann, "LISA pathfinder," Classical Quantum Gravity 25, 114034 (2008)

${ }^{3}$ F. Antonucci, M. Armano, H. Audley, G. Auger, M. Benedetti, P. Binetruy, C. Boatella, J. Bogenstahl, D. Bortoluzzi, P. Bosetti, N. Brandt, M. Caleno, A. Cavalleri, M. Cesa, M. Chmeissani, G. Ciani, A. Conchillo, G. Congedo, I. Cristofolini, M. Cruise, K. Danzmann, F. De Marchi, M. Diaz-Aguilo, I. Diepholz, G. Dixon, R. Dolesi, N. Dunbar, J. Fauste, L. Ferraioli, D. Fertin, W. Fichter, E. Fitzsimons, M. Freschi, A. García Marin, C. G. Marirrodriga, R. Gerndt, L. Gesa, D. Giardini, F. Gibert, C. Grimani, A. Grynagier, B. Guillaume, F. Guzmán, I. Harrison, G. Heinzel, M. Hewitson, D. Hollington, J. Hough, D. Hoyland, M. Hueller, J. Huesler, O. Jeannin, O. Jennrich, P. Jetzer, B. Johlander, C. Killow, X. Llamas, I. Lloro, A. Lobo, R. Maarschalkerweerd, S. Madden, D. Mance, I. Mateos, P. W. McNamara, J. Mendes, E. Mitchell, A. Monsky, D. Nicolini, D. Nicolodi, M. Nofrarias, F. Pedersen, M. Perreur-Lloyd, A. Perreca, E. Plagnol, P. Prat, G. D. Racca, B. Rais, J. Ramos-Castro, J. Reiche, J. A. R. Perez, D. Robertson, H. Rozemeijer, J. Sanjuan, A. Schleicher, M. Schulte, D. Shaul, L. Stagnaro, S. Strandmoe, F. Steier, T. J. Sumner, A. Taylor, D. Texier, C. Trenkel, D. Tombolato, S. Vitale, G. Wanner, H. Ward, S. Waschke, P. Wass, W. J. Weber, and P. Zweifel, "From laboratory experiments to LISA pathfinder: Achieving LISA geodesic motion," Classical Quantum Gravity 28, 094002 (2011).

${ }^{4}$ F. Antonucci, M. Armano, H. Audley, G. Auger, M. Benedetti, P. Binetruy, C. Boatella, J. Bogenstahl, D. Bortoluzzi, P. Bosetti, M. Caleno, A. Cavalleri, M. Cesa, M. Chmeissani, G. Ciani, A. Conchillo, G. Congedo, I. Cristofolini, M. Cruise, K. Danzmann, F. De Marchi, M. Diaz-Aguilo, I. Diepholz, G. Dixon, R. Dolesi, N. Dunbar, J. Fauste, L. Ferraioli, D. Fertin, W. Fichter, E. Fitzsimons, M. Freschi, A. G. Marin, C. G. Marirrodriga, R. Gerndt, L. Gesa, F. Gilbert, D. Giardini, C. Grimani, A. Grynagier, B. Guillaume, F. Guzmán, I. Harrison, G. Heinzel, M. Hewitson, D. Hollington, J. Hough, D. Hoyland, M. Hueller, J. Huesler, O. Jeannin, O. Jennrich, P. Jetzer, B. Johlander, C. Killow, X. Llamas, I. Lloro, A. Lobo, R. Maarschalkerweerd, S. Madden, D. Mance, I. Mateos, P. W. McNamara, J. Mendes, E. Mitchell, A. Monsky, D. Nicolini, D. Nicolodi, M. Nofrarias, F. Pedersen, M. Perreur-Lloyd, A. Perreca, E. Plagnol, P. Prat, G. D. Racca, B. Rais, J. Ramos-Castro, J. Reiche, J. A. R. Perez, D. Robertson, H. Rozemeijer, J. Sanjuan, A. Schleicher, M. Schulte, D. Shaul, L. Stagnaro, S. Strandmoe, F. Steier, T. J. Sumner, A. Taylor, D. Texier, C. Trenkel, D. Tombolato, S. Vitale, G. Wanner, H. Ward, S. Waschke, P. Wass, W. J. Weber, and P. Zweifel, "LISA pathfinder: Mission and status," Classical Quantum Gravity 28, 094001 (2011).

${ }^{5}$ F. Antonucci, M. Armano, H. Audley, G. Auger, M. Benedetti, P. Binetruy, J. Bogenstahl, D. Bortoluzzi, P. Bosetti, N. Brandt, M. Caleno, P. Cañizares, A. Cavalleri, M. Cesa, M. Chmeissani, A. Conchillo, G. Congedo, I. Cristofolini, M. Cruise, K. Danzmann, F. De Marchi, M. Diaz-Aguilo, I. Diepholz, G. Dixon, R. Dolesi, N. Dunbar, J. Fauste, L. Ferraioli, V. Ferrone, W. Fichter, E. Fitzsimons, M. Freschi, A. G. Marin, C. G. Marirrodriga, R. Gerndt, L. Gesa, F. Gilbert, D. Giardini, C. Grimani, A. Grynagier, B. Guillaume, F. Guzmán, I. Harrison, G. Heinzel, V. Hernández, M. Hewitson, D. Hollington, J. Hough, D. Hoyland, M. Hueller, J. Huesler, O. Jennrich, P. Jetzer, B. Johlander, N. Karnesis, C. Killow, X. Llamas, I. Lloro, A. Lobo, R. Maarschalkerweerd, S. Madden, D. Mance, I. Mateos, P. W. McNamara, J. Mendes, E. Mitchell, A. Monsky, D. Nicolini, D. Nicolodi, M. Nofrarias, F. Pedersen, M. Perreur-Lloyd, E. Plagnol, P. Prat, G. D. Racca, J. Ramos-Castro, J. Reiche, J. A. R. Perez, D. Robertson, H. Rozemeijer, J. Sanjuan, A. Schleicher, M. Schulte, D. Shaul, L. Stagnaro, S. Strandmoe, F. Steier, T. J. Sumner, A. Taylor, D. Texier, C. Trenkel, H.-B. Tu, S. Vitale, G. Wanner, H. Ward, S. Waschke, P. Wass, W. J. Weber, T. Ziegler, and P. Zweifel, “The LISA pathfinder mission," Classical Quantum Gravity 29, 124014 (2012).
${ }^{6}$ M. Armano, H. Audley, G. Auger, J. Baird, M. Bassan, P. Binetruy, M. Born, D. Bortoluzzi, N. Brandt, M. Caleno, L. Carbone, A. Cavalleri, A. Cesarini, G. Ciani, G. Congedo, A. Cruise, K. Danzmann, M. de Deus Silva, R. De Rosa, M. Diaz-Aguiló, L. Di Fiore, I. Diepholz, G. Dixon, R. Dolesi, N. Dunbar, L. Ferraioli, V. Ferroni, W. Fichter, E. Fitzsimons, R. Flatscher, M. Freschi, A. García Marín, C. García Marirrodriga, R. Gerndt, L. Gesa, F. Gibert, D. Giardini, R. Giusteri, F. Guzmán, A. Grado, C. Grimani, A. Grynagier, J. Grzymisch, I. Harrison, G. Heinzel, M. Hewitson, D. Hollington, D. Hoyland, M. Hueller, H. Inchauspé, O. Jennrich, P. Jetzer, U. Johann, B. Johlander, N. Karnesis, B. Kaune, N. Korsakova, C. Killow, J. Lobo, I. Lloro, L. Liu, J. LópezZaragoza, R. Maarschalkerweerd, D. Mance, V. Martín, L. Martin-Polo, J. Martino, F. Martin-Porqueras, S. Madden, I. Mateos, P. McNamara, J. Mendes, L. Mendes, A. Monsky, D. Nicolodi, M. Nofrarias, S. Paczkowski, M. PerreurLloyd, A. Petiteau, P. Pivato, E. Plagnol, P. Prat, U. Ragnit, B. Raïs, J. RamosCastro, J. Reiche, D. Robertson, H. Rozemeijer, F. Rivas, G. Russano, J. Sanjuán, P. Sarra, A. Schleicher, D. Shaul, J. Slutsky, C. Sopuerta, R. Stanga, F. Steier, T. Sumner, D. Texier, J. Thorpe, C. Trenkel, M. Tröbs, H. Tu, D. Vetrugno, S. Vitale, V. Wand, G. Wanner, H. Ward, C. Warren, P. Wass, D. Wealthy, W. Weber, L. Wissel, A. Wittchen, A. Zambotti, C. Zanoni, T. Ziegler, and P. Zweifel, "Sub-femto-g free fall for space-based gravitational wave observatories: LISA pathfinder results," Phys. Rev. Lett. 116, 231101 (2016).

${ }^{7}$ M. Armano, H. Audley, J. Baird, P. Binetruy, M. Born, D. Bortoluzzi, E. Castelli, A. Cavalleri, A. Cesarini, A. Cruise, K. Danzmann, M. de Deus Silva, I. Diepholz, G. Dixon, R. Dolesi, L. Ferraioli, V. Ferroni, E. Fitzsimons, M. Freschi, L. Gesa, F. Gibert, D. Giardini, R. Giusteri, C. Grimani, J. Grzymisch, I. Harrison, G. Heinzel, M. Hewitson, D. Hollington, D. Hoyland, M. Hueller, H. Inchauspé, O. Jennrich, P. Jetzer, N. Karnesis, B. Kaune, N. Korsakova, C. Killow, J. Lobo, I. Lloro, L. Liu, J. López-Zaragoza, R. Maarschalkerweerd, D. Mance, N. Meshksar, V. Martín, L. Martin-Polo, J. Martino, F. Martin-Porqueras, I. Mateos, P. McNamara, J. Mendes, L. Mendes, M. Nofrarias, S. Paczkowski, M. Perreur-Lloyd, A. Petiteau, P. Pivato, E. Plagnol, J. Ramos-Castro, J. Reiche, D. Robertson, F. Rivas, G. Russano, J. Slutsky, C. Sopuerta, T. Sumner, D. Texier, J. Thorpe, D. Vetrugno, S. Vitale, G. Wanner, H. Ward, P. Wass, W. Weber, L. Wissel, A. Wittchen, and P. Zweifel, "Beyond the required LISA free-fall performance: New LISA pathfinder results down to $20 \mu \mathrm{Hz}$," Phys. Rev. Lett. 120, 061101 (2018). ${ }^{8} \mathrm{D}$. Mance, "Development of electronic system for sensing and actuation of test mass of the inertial sensor LISA," Thesis, University of Split, Croatia, 2012, available at http://spaceserv1.ethz.ch/aeil/download/Davor_Mance_Thesis_ text.pdf.

${ }^{9}$ M. Armano, H. Audley, G. Auger, J. Baird, M. Bassan, P. Binetruy, M. Born, D. Bortoluzzi, N. Brandt, M. Caleno, A. Cavalleri, A. Cesarini, A. Cruise, K. Danzmann, M. de Deus Silva, R. De Rosa, L. Di Fiore, I. Diepholz, G. Dixon, R. Dolesi, N. Dunbar, L. Ferraioli, V. Ferroni, E. Fitzsimons, R. Flatscher, M. Freschi, C. García Marirrodriga, R. Gerndt, L. Gesa, F. Gibert, D. Giardini, R. Giusteri, A. Grado, C. Grimani, J. Grzymisch, I. Harrison, G. Heinzel, M. Hewitson, D. Hollington, D. Hoyland, M. Hueller, H. Inchauspé, O. Jennrich, P. Jetzer, B. Johlander, N. Karnesis, B. Kaune, N. Korsakova, C. Killow, J. Lobo, I. Lloro, L. Liu, J. López-Zaragoza, R. Maarschalkerweerd, D. Mance, V. Martín, L. MartinPolo, J. Martino, F. Martin-Porqueras, S. Madden, I. Mateos, P. McNamara, J. Mendes, L. Mendes, N. Meshksar, M. Nofrarias, S. Paczkowski, M. PerreurLloyd, A. Petiteau, P. Pivato, E. Plagnol, P. Prat, U. Ragnit, J. Ramos-Castro, J. Reiche, D. Robertson, H. Rozemeijer, F. Rivas, G. Russano, P. Sarra, A. Schleicher, J. Slutsky, C. Sopuerta, R. Stanga, T. Sumner, D. Texier, J. Thorpe, C. Trenkel, M. Tröbs, D. Vetrugno, S. Vitale, G. Wanner, H. Ward, P. Wass, D. Wealthy, W. Weber, L. Wissel, A. Wittchen, A. Zambotti, C. Zanoni, T. Ziegler, and P. Zweifel, "Capacitive sensing of test mass motion with nanometer precision over millimeter-wide sensing gaps for space-borne gravitational reference sensors," Phys. Rev. D 96, 062004 (2017).

${ }^{10}$ W. J. Weber, D. Bortoluzzi, A. Cavalleri, L. Carbone, M. D. Lio, R. Dolesi, G. Fontana, C. D. Hoyle, M. Hueller, and S. Vitale, "Position sensors for flight testing of LISA drag-free control," Proc. SPIE 4856, 31-42 (2003).

${ }^{11}$ R. Dolesi, D. Bortoluzzi, P. Bosetti, L. Carbone, A. Cavalleri, I. Cristofolini, M. DaLio, G. Fontana, V. Fontanari, B. Foulon, C. D. Hoyle, M. Hueller, F. Nappo, P. Sarra, D. N. A. Shaul, T. Sumner, W. J. Weber, and S. Vitale, "Gravitational sensor for LISA and its technology demonstration mission," Classical Quantum Gravity 20, S99-S108 (2003). 
${ }^{12}$ W. J. Weber, "Nano-Newton electrostatic actuators for femto-Newton smallforce measurements: Performance of the LISA pathfinder electrostatic force actuation system" (unpublished)

${ }^{13}$ See www.analog.com for Analog Devices; accessed 2000-2007.
${ }^{14} \mathrm{~N}$. Meshksar, L. ferraioli, D. Mance, and J. ten Pierick, "Electrostatic actuation accuracy for the laser interferometer space antenna (LISA)" (unpublished).

${ }^{15}$ MATLAB, version 9.3.0.713579 (R2017b), The MathWorks Inc., Natick, Massachusetts, 2017. 